\title{
Effect of Residual Maleic Anhydride in PP-g-MAH on the Thermo-Oxidative Aging Properties of RGF-PP
}

\author{
Junjie Tang1 ${ }^{*}$, Pengwei Shi', Hao Duan'1, Kwang-Yoon Kim¹, Minqi Xin ${ }^{2}$ \\ ${ }^{1}$ Fine-Blend Compatilizer Jiangsu Co., Ltd., Shanghai, China \\ ${ }^{2}$ Shanghai Kumho Sunny Plastics Co., Ltd., Shanghai, China \\ Email: *tangjunjie@fineblend.com.cn
}

How to cite this paper: Tang, J.J., Shi, P.W., Duan, H., Kim, K.-Y. and Xin, M.Q. (2017) Effect of Residual Maleic Anhydride in PP-g-MAH on the Thermo-Oxidative Aging Properties of RGF-PP. Journal of Materials Science and Chemical Engineering, 5, 61-70.

https://doi.org/10.4236/msce.2017.57008

Received: May 8, 2017

Accepted: July 9, 2017

Published: July 13, 2017

Copyright $\odot 2017$ by authors and Scientific Research Publishing Inc. This work is licensed under the Creative Commons Attribution International License (CC BY 4.0).

http://creativecommons.org/licenses/by/4.0/

c) (i) Open Access

\begin{abstract}
The mechanical, morphological and thermo-oxidative aging properties of the glass fiber reinforced polypropylene (RGF-PP) were studied based on four different maleic anhydride grafted polypropylene (PP- $g$-MAH) compatibilizers with different content of residual maleic anhydride (MAH). It was shown that PP-g-MAH compatibilizer could significantly improve the mechanical properties of RGF-PP, while from thermal and morphological analysis results, the content of residual MAH had negative influence on long term thermooxidative aging properties of RGF-PP with adding PP- $g$-MAH compatibilizer; the lower the residual content of MAH is, the better the thermo stability of the $\mathrm{PP}-\mathrm{g}$-MAH is, and also the better the thermo-oxidative aging properties was obtained.
\end{abstract}

\section{Keywords}

Residual Maleic Anhydride, Maleic Anhydride Grafted Polypropylene, RGF-PP, Thermo-Oxidative Aging

\section{Introduction}

Polypropylene (PP) has advantages of low density, cheap price, good processing property and chemical resistance as a general-purpose of plastics so that it is widely applied in the field of household appliances, automobiles, packaging and construction. On the other hand, various disadvantages of it such as low mechanical property, high molding shrinkage, low impact strength near $\mathrm{T}_{\mathrm{g}}$ and low distortion temperature limited the use of PP [1]. The modification and processing technologies of PP was developed in order to make up the drawback of PP and the RGF-PP was one of the applicable ways for enhancement of mechan- 
ical property and finally replaced for engineering plastics.

The needs of requirements for the properties of RGF-PP were raised upon expanded application. For example, the parts used in engines need to work for a long time at $100^{\circ} \mathrm{C}$, which means that the RGF-PP has to possess better thermo-oxidative aging properties. In general, when $\mathrm{PP}$ is exposed to thermodynamic field for a long time, the covalent $\mathrm{C}-\mathrm{C}$ or $\mathrm{C}-\mathrm{H}$ bond will break and form radicals, resulting in reducing the molecular weight of $\mathrm{PP}$ due to a degradation on a macro level [2].

To evaluate the thermo-oxidative aging properties, the artificial accelerated aging test was used. The materials were stored in an oven for a certain period at a given temperature [3].

In general, PP and glass fiber or other minerals have poor compatibility. In order to improve the interfacial bonding strength between PP and glass fiber or other minerals, maleic anhydride grafted polypropylene (PP-g-MAH) is often used as a compatibilizer [4] [5] [6] [7] [8]. Thus, the grafting content of MAH in $\mathrm{PP}-\mathrm{g}$-MAH is one of the important factors for improved property of PP, though most commercial PP- $g$-MAH compatibilizer has proper grafting content used in RGF-PP at present. On the other hand, residual MAH exist in PP-g-MAH is another important parameter affecting the property of RGF-PP, especially the thermo-oxidative aging property of the composite. However, few people have concerned about it.

Thus, in this paper, the effect of different residual contents of MAH in PP- $g$-MAH on the thermo-oxidative aging properties of the RGF-PP is studied in terms of the tensile strength, notched impact strength and phase morphology.

\section{Experiment}

Materials. PP was supplied by SECCO Petrochemical Co., Ltd. (Shanghai, China) with the trade name of K7926. Alkali-free glass fiber (trade name: 988A) was purchased from Jushi group, Tongxiang, China. The compatibilizer, PP- $g$-MAH, which was the trade name of CMG9801, was supplied by Fine-Blend Compatilizer Jiangsu Co., Ltd. (Shanghai, China). Another Commercial PP-g-MAH compatibilizer $\mathrm{A}, \mathrm{B}$ and $\mathrm{C}$ were purchased from the market. Three different antioxidants, 1010 which is pentaerythritol tetrakys 3-(3, 5-ditert-butyl-4-hydroxyphenyl) propionate, 168 which is tri (2, 4-di-tert-butylphenyl) phosphite and DSTP which is (dioctadecyl 3, 3'-thiodipropionate) were all purchased from Ciba, Switzerland.

Sample preparation. The samples were prepared by melt extrusion using a Ф36-diameter twin-screw extruder (SHJ-36) at $300 \mathrm{rpm}$. The barrel temperatures were fixed at $210^{\circ} \mathrm{C}-220^{\circ} \mathrm{C}$. The particles were dried at $100^{\circ} \mathrm{C}$ for $3 \mathrm{~h}$ and injection molded into the ASTM standard samples at the charging barrel temperature of $230^{\circ} \mathrm{C}$ using HTF80X1 machine. The various samples were numbered according to the following components and ingredients listed in Table 1.

Test for mechanical properties. The electronic universal testing machine (CMT6104) and impact test machine (XC-2.75) were used to test the tensile and 
Table 1. Test formula of RGF-PP.

\begin{tabular}{cccccc}
\hline Component & $1 \#$ & $2 \#$ & $3 \#$ & $4 \#$ & $5 \#$ \\
\hline PP & 70 & 67 & 67 & 67 & 67 \\
Glass fiber & 30 & 30 & 30 & 30 & 30 \\
CMG9801 & & 3 & & & \\
Compatibilizer A & & & 3 & & \\
Compatibilizer B & & & & 3 & \\
Compatibilizer C & & & & & 3 \\
1010 & 0.2 & 0.2 & 0.2 & 0.2 & 0.2 \\
168 & 0.2 & 0.2 & 0.2 & 0.2 & 0.2 \\
DSTP & 0.4 & 0.4 & 0.4 & 0.4 & 0.4 \\
\hline
\end{tabular}

notched impact strength of the samples, respectively. The tensile strength and notched impact strength were tested according to the ASTM D638-2008 and D256a-2006, respectively.

Purification and determination of grafting content of PP-g-MAH. The four different PP- $g$-MAH compatibilizers were dissolved in refluxing xylene and the excess acetone was added to precipitate them. Then, the precipitates were filtered, washed and dried under vacuum at $80^{\circ} \mathrm{C}$ for $24 \mathrm{~h}$.

The grafting content of purified PP- $g$-MAH was determined by a nonaqueous back-titration. Some amount of purified PP-g-MAH was dissolved in hot xylene, followed by the addition of excess potassium hydroxide-ethanol standard solution in xylene. The solution was kept refluxing, and then titrated with hydrochloric acid-isopropanol standard solution using phenolphthalein as an indicator.

The equation to calculate the grafting content of PP- $g$-MAH can be expressed as:

$$
G_{M A H}=9.806 \times\left(C_{1} V_{1}-C_{2} V_{2}\right) / 2 m
$$

where $G_{M A H}(\%)$ represents the grafting content of PP-g-MAH. $C_{1}(\mathrm{~mol} / \mathrm{L})$ and $C_{2}(\mathrm{~mol} / \mathrm{L})$ are the molar concentration of potassium hydroxide-ethanol standard solution and hydrochloric acid-isopropanol standard solution, respectively. $V_{1}(\mathrm{~mL})$ and $V_{2}(\mathrm{~mL})$ are the volume of potassium hydroxide-ethanol standard solution and hydrochloric acid-isopropanol standard solution, respectively. $m$ represents the weight of the purified PP- $g$-MAH and 98.06 represents the molecular weight of MAH [9].

Determination the residual content of MAH in PP-g-MAH. The total content of MAH of rough PP-g-MAH was also determined by the nonaqueous back-titration under the same conditions as above.

The equation to calculate the total MAH content of rough PP-g-MAH can be expressed as:

$$
G^{\prime}=9.806 \times\left(C_{1} V_{1}^{\prime}-C_{2} V_{2}^{\prime}\right) / 2 m
$$

where $G^{\prime}(\%)$ represents the total MAH content of PP- $g$-MAH. $C_{1}(\mathrm{~mol} / \mathrm{L})$ and $C_{2}(\mathrm{~mol} / \mathrm{L})$ are the molar concentration of potassium hydroxide-ethanol 
standard solution and hydrochloric acid-isopropanol standard solution, respectively. $V_{1}^{\prime}(\mathrm{mL})$ and $V_{2}^{\prime}(\mathrm{mL})$ are the volume of potassium hydroxide-ethanol standard solution and hydrochloric acid-isopropanol standard solution, respectively. $m$ represents the weight of the rough $\mathrm{PP}-g$-MAH and 98.06 represents the molecular weight of MAH.

The equation to calculate residual content of MAH in PP-g-MAH can be expressed as:

$$
R=G^{\prime}-G_{M A H}
$$

where $R(\%)$ is the residual content of MAH in rough PP-g-MAH, $G^{\prime}$ (\%) is the total MAH content in rough PP-g-MAH, and $G_{M A H}(\%)$ is the grafting content of purified PP- $g$-MAH [10].

Thermal analysis. The PP-g-MAH compatibilizers were measured using a NETZSCH thermogravimetric analysis (TG $209 \mathrm{~F} 1$ ) from room temperature to $500^{\circ} \mathrm{C}$ under nitrogen atmosphere at a heating rate of $10^{\circ} \mathrm{C} / \mathrm{min}$.

Morphological Observation. The freeze-fractured surface of the RGF-PP samples at the liquid nitrogen temperature was used to observe the phase morphology using a Hitachi S-4800 scanning electron microscope (SEM). The surface was coated with gold and the accelerating voltage was $25 \mathrm{kV}$.

Thermo-oxidative aging test. The RGF-PP samples were put in aging test chamber (HOC-HQ60) at constant temperature, $150^{\circ} \mathrm{C}$ at various aging periods of $250,500,900$ and $1200 \mathrm{~h}$. The aged samples were stored at room temperature for $16 \mathrm{~h}$ and the tests were carried out for mechanical properties.

\section{Results and Discussion}

The grafting content and residual content of MAH in different PP-g-MAH. As shown in Table 2, the grafting content and residual content of MAH from four commercial PP-g-MAH compatibilizers were calculated by nonaqueous back-titration. The grafting content of CMG9801, A, B and C was $0.98 \%, 0.85 \%$, $0.92 \%$ and $0.79 \%$, respectively. In addition, the residual content of $\mathrm{MAH}$ was $0.25 \%, 0.56 \%, 0.57 \%$ and $0.24 \%$, respectively. Thus, CMG9801 has the highest grafting content and almost the lowest residual content of MAH among the four compatibilizers.

The effect of compatibilizers on the mechanical properties of RGF-PP before aging. Figure 1 shows the tensile strength and impact strength of samples from $1 \#$ to $5 \#$. The tensile strength of sample $1 \#$ was about $30 \mathrm{MPa}$, while

Table 2. The comparison of the grafting content and residual content of MAH for different PP-g-MAH compatibilizers.

\begin{tabular}{ccccc}
\hline Compati-Bilizers & CMG 9801 & Compatibilizer A & Compatibilizer B & Compatibilizer C \\
\hline Grafting content /\% & 0.98 & 0.85 & 0.92 & 0.79 \\
Total MAH content /\% & 1.23 & 1.41 & 1.49 & 1.03 \\
Residual content of MAH/\% & 0.25 & 0.56 & 0.57 & 0.24 \\
\hline
\end{tabular}




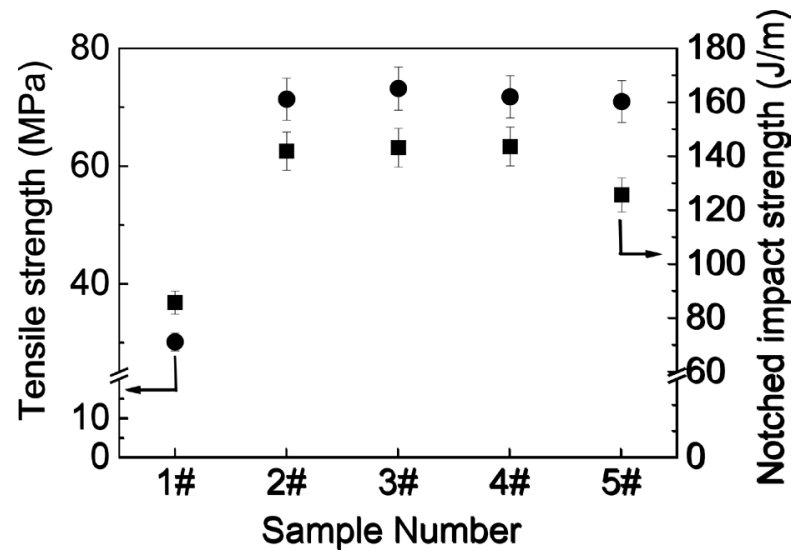

Figure 1. The mechanical properties of RGF-PP adding different PP-g-MAH; tensile strength $(\bullet)$, notched impact strength $(\bullet)$.

that of samples 2\# - 5\# was dramatically increased to $71-73 \mathrm{MPa}$, which meant that the effective binding force between PP- $g$-MAH and RGF-PP matrix was not formed without compatibilizer as seen in sample $1 \#$. In addition, no particular difference of each compatibilizer between CMG9801 and A, B or C was obtained, resulting in the role of PP-g-MAH was similar in the RGF-PP system. The above experimental data implies us that PP- $g$-MAH play an important role in improving the tensile strength of the RGF-PP matrix.

For the notched impact strength, that of sample $1 \#$ was $84 \mathrm{~J} / \mathrm{m}$, that of samples 2\# - 4\# was 145 - $147 \mathrm{~J} / \mathrm{m}$, and that of sample 5\# was about $125 \mathrm{~J} / \mathrm{m}$. The notched impact strength without compatibilizer (sample1\#) showed poor interfacial interaction, while that with compatibilizer C (sample 5\#) was lower than others, Thus, it was suggested that compatibilizer $C$ show the inferior impact strength due to the lower grafting content of the PP-g-MAH, resulting in for formation of poor interfacial bonding energy between PP and glass fiber was not good enough.

The effect of compatibilizers on the mechanical properties of RGF-PP at various aging periods.

The tensile strength and notched impact strength of samples $2 \#$ - 5\# measured after aging at various periods such as 250, 500, 900 and $1200 \mathrm{~h}$, were plotted in Figure 2 and Figure 3, respectively.

As seen in Figure 2, the tensile strength of samples 2\# slightly increased with aging periods, while those value of $3 \#-5 \#$ were similar up to $500 \mathrm{~h}$, but dramatically decreased upon further aging periods. On the other hand, the notched impact strength of samples $2 \#$ and 5\# slightly decreased upon aging periods. Where as those of 3\# and 4\# dramatically dropped after $500 \mathrm{~h}$ as shown in Figure 3. The difference between samples $2 \#, 5 \#$ and samples $3 \#, 4 \#$ is the residual content of MAH in different compatibilizers, where the former contains $0.25 \%$ and $0.24 \%$ and the latter contains $0.56 \%$ and $0.57 \%$, respectively. Thus, the main cause of poor mechanical properties upon aging would be large contents of residual MAH in compatibilizers B and C.

The thermal properties of different PP-g-MAH. It was studied that the free 


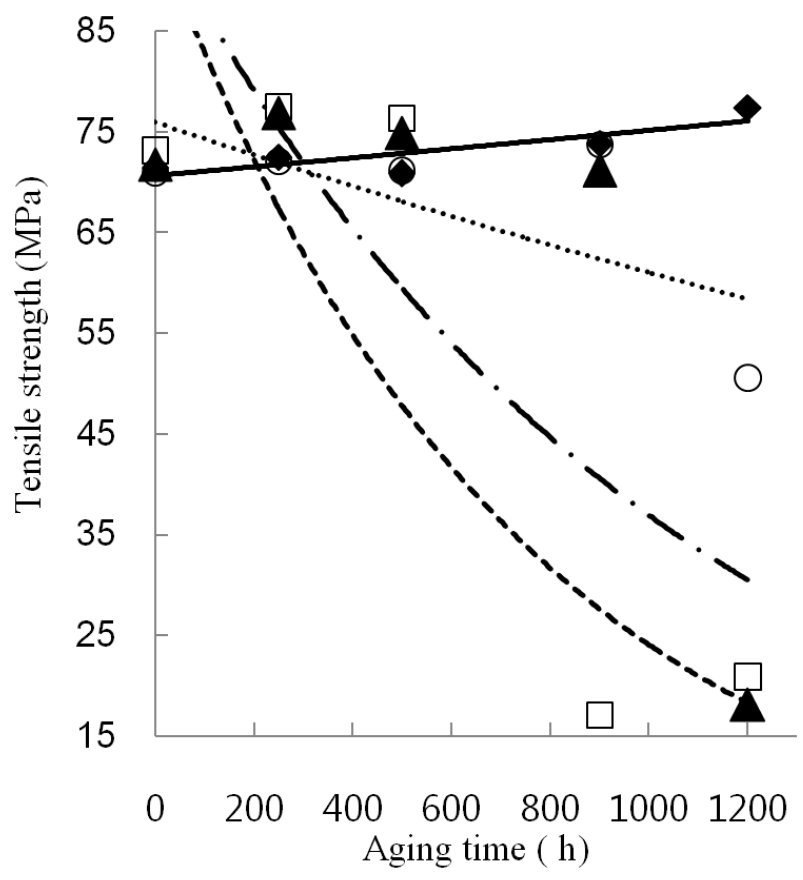

Figure 2. Effect of PP- $g$-MAH on the tensile strength of RGF-PP at various aging periods; $(\diamond$ : Sample 2\#, $\square$ : Sample 3\#, $\boldsymbol{\Lambda}$ : Sample 4\#, ○: Sample 5\#; — CMG9801; ..... Compatibilizer A; •- Compatibilizer B; ----- Compatibilizer C).

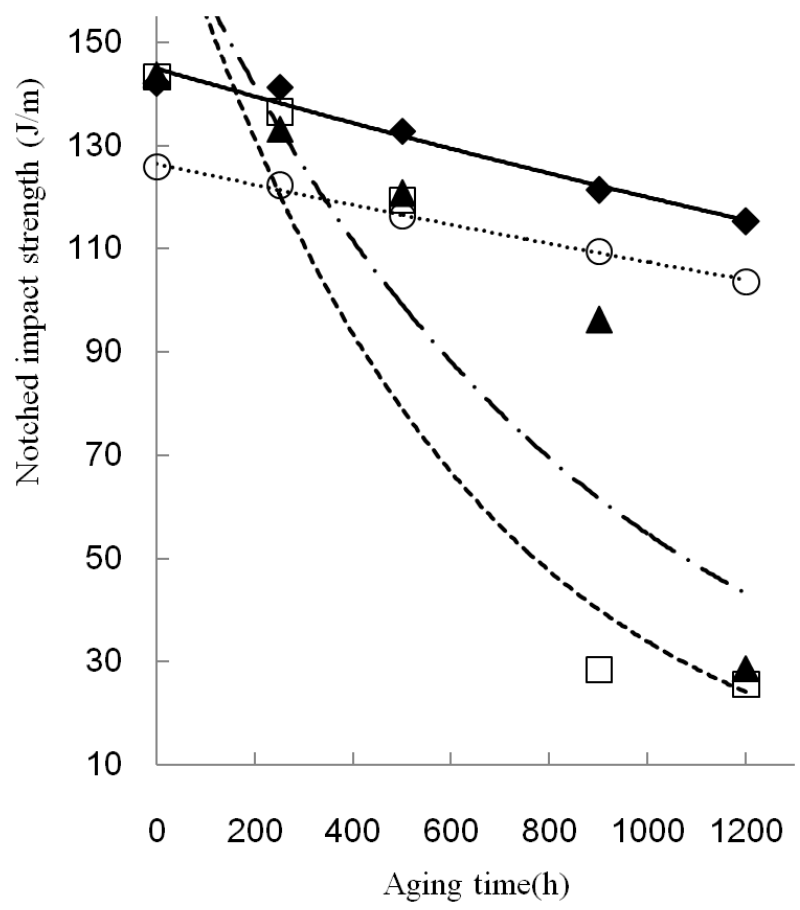

Figure 3. Effect of PP- $g$-MAH on the notched strength of RGF-PP at various aging periods; $(\diamond:$ Sample 2\#, 口: Sample 3\#, $\boldsymbol{\Delta}$ : Sample 4\#, ○: Sample 5\#; - CMG9801, ..... Compatibilizer A, •-Compatibilizer B, ----- Compatibilizer C).

MAH residue influenced on the thermal stability of PP- $g$-MAH. For the PP- $g$ MAH compatibilizer with high free MAH residue, the initial decomposition temperature occurred earlier, while the high residue had few effects on decom- 
position speed in regards to the quick volatilization of free MAH at high temperature [11]. The conclusion of this paper matched with the former studies as shown in Table 3 and Figure 4.

From Table 3 and Figure 4, the thermal decomposition temperature of CMG9801 was higher than compatibilizer A and B, while similar to compatibilizer C. Especially in initial decomposition stage, the thermal decomposition temperatures of CMG9801 and compatibilizer $\mathrm{C}$ was $10^{\circ} \mathrm{C}$ higher than that of compatibilizer $\mathrm{A}$ and $\mathrm{B}$. This tell us that more $\mathrm{MAH}$ residue in compatibilizer $\mathrm{A}$ and $\mathrm{B}$ affected the decomposition temperature at initial stage and then affected the thermo-oxidative aging properties of RGF-PP at high temperature.

The effect of aging time on the morphology. Figures 5(a)-(e) showed the SEM photographs of samples 1\# - 5\# before aging; As seen in Figure 5(a), the discrete surface between PP and glass fiber was observed due to the absence of compatibilizer, implying that no binding force was formed so that the mechanical properties would be poor as shown in Figure 1. Meanwhile, as seen in Figures 5(b)-(e) represent samples from 2\# to 5\#, by adding CMG9801, compatibilizer $\mathrm{A}, \mathrm{B}$ and $\mathrm{C}$, respectively, the morphology of the interfacial binding force between PP and glass fiber seemed to be induced. This would develop the improved tensile strength and notched impact strength as shown in Figure 1.

While in Figures 5(a')-(e'), it represented the SEM photographs of the interface between PP and glass fiber for samples from 1\# to 5\# after aging for $1200 \mathrm{~h}$ at $150^{\circ} \mathrm{C}$. The interface between PP and glass fiber matrix were destroyed, and pulverization phenomenon was observed in the PP matrix of samples 1\#, 3\# and

Table 3. Thermal decomposition parameters of different PP-g-MAH.

\begin{tabular}{ccccc}
\hline \multirow{2}{*}{ Compatibilizers } & \multicolumn{3}{c}{ Decomposition temperature } \\
\cline { 2 - 4 } & $5 \%$ weight loss & 10\% weight loss & 20\% weight loss & 50\% weight loss \\
\hline CMG9801 & 394.9 & 418.9 & 435.4 & 454.4 \\
Compatibilizer A & 384.9 & 408.4 & 427.9 & 448.9 \\
Compatibilizer B & 390.3 & 410.3 & 426.8 & 442.6 \\
Compatibilizer C & 397.3 & 419.8 & 435.8 & 454.3 \\
\hline
\end{tabular}

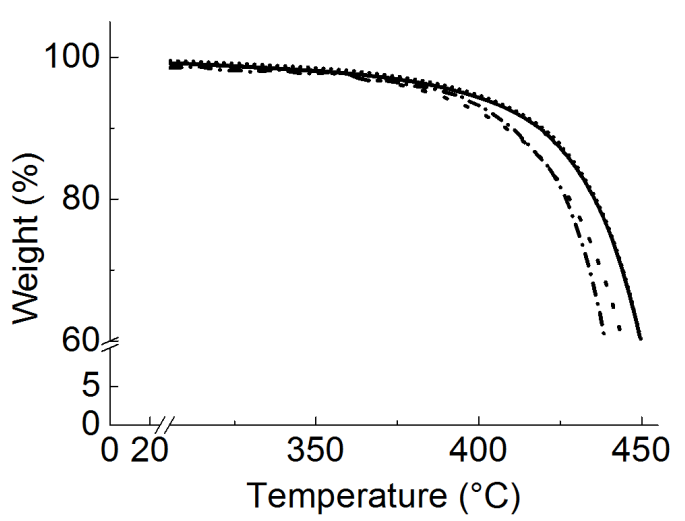

Figure 4. TGA curves of different PP-g-MAH compatibilizers; ( - CMG9801, ••••

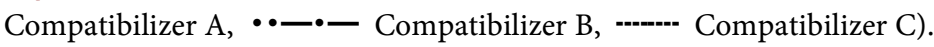



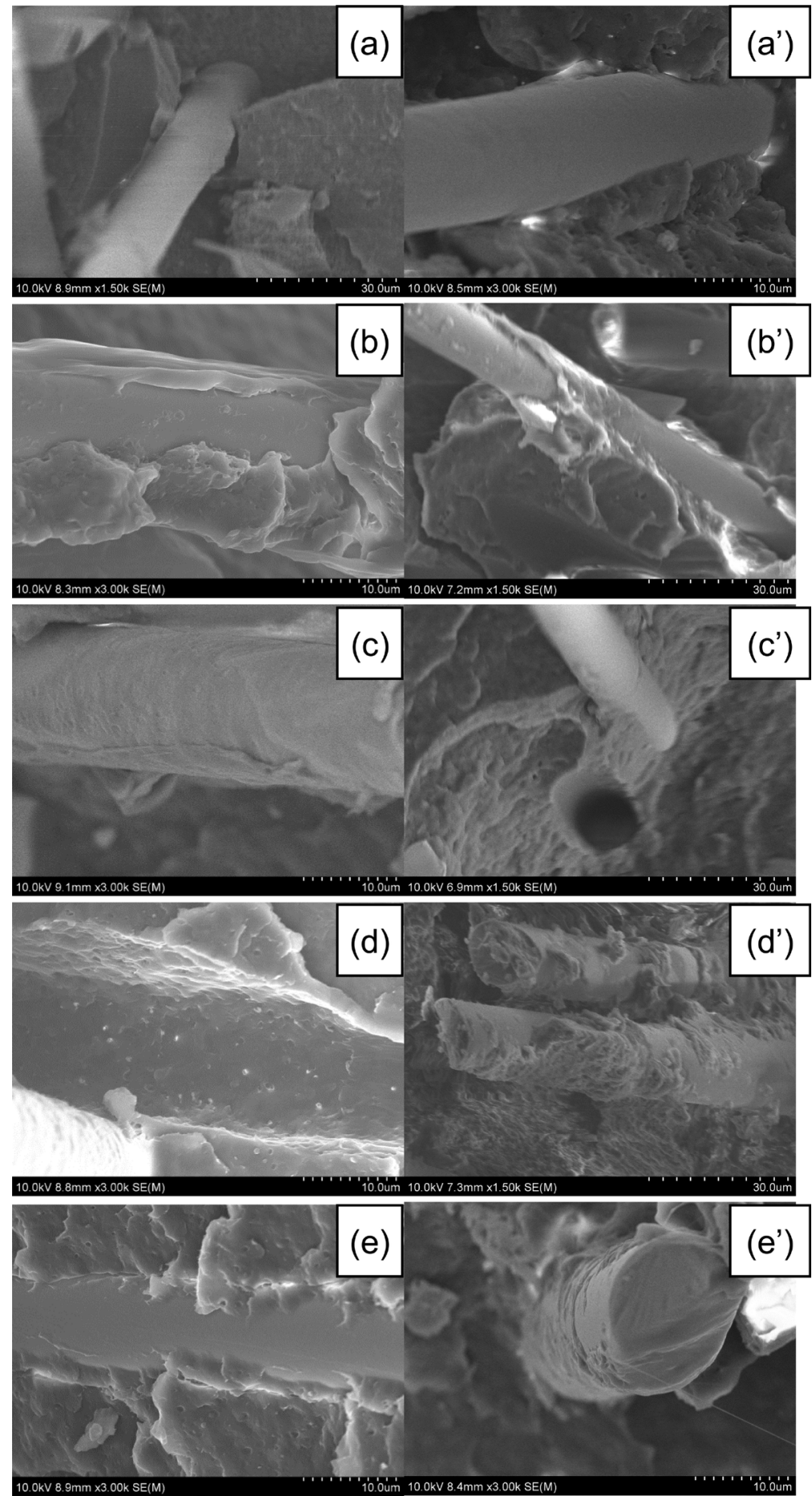

Figure 5. SEM photographs of the samples $1 \#-5 \#$ before aging and after aging $1200 \mathrm{~h}$ at $150^{\circ} \mathrm{C}$; (a) 1\#, (b) 2\#, (c) 3\#, (d) $4 \#$ and (e) $5 \#$ for the samples before aging, (a') 1\#, (b') 2\#, (c') 3\#, (d') $4 \#$ and (e') $5 \#$ for the samples after aging $1200 \mathrm{~h}$ at $150^{\circ} \mathrm{C}$.

4\#; while the interface of that was maintained in good shape upon adding CMG9801 and compatibilizer C. This phenomenon may induce that the sample 2\# and 5\# retained the higher mechanical properties as shown in Figure 2 and Figure 3.

In conclusion, from the view of mechanical properties and SEM photographs 
before aging, the difference of mechanical properties of RGF-PP was not so obvious because of adding PP- $g$-MAH compatibilizers which had proper grafting content. While after aging for $1200 \mathrm{~h}$ for $150^{\circ} \mathrm{C}$, those samples adding high $\mathrm{MAH}$ residue content PP- $g$-MAH just like compatibilizer A and B had poor mechanical properties and morphology, that is because too much free MAH in compatibilizer can destroy the PP molecular chain structure at high temperature for a long time to promote the PP chain decomposition and decrease the mechanical properties. All in all, the main reason of affecting the long-term thermo-oxidative aging properties of RGF-PP materials was the MAH residue content with adding PP- $g$-MAH compatibilizer.

\section{Conclusions}

1) For RGF-PP materials, the mechanical properties improved with adding PP- $g$-MAH compatibilizer.

2) For PP-g-MAH compatibilizers, the residual MAH had effect on thermal properties of PP-g-MAH compatibilizer. The lower the residual content of $\mathrm{MAH}$, the better the thermal stability of the compatibilizer could be achieved.

3) For RGF-PP materials, the amount of MAH residue had effective influence on long-term thermo-oxidative aging properties with adding PP-g-MAH compatibilizer. The lower the residual content of MAH, the better the thermooxidative aging properties were obtained.

\section{References}

[1] Yilser, G., Sinan, E., Zakir, M.O.R. and Erhan, P. (2004) Comparison of Maleic Anhydride Grafting onto Powder and Granular Polypropylene in the Melt by Reactive Extrusion. Journal of Applied Polymer Science, 3675, 92.

[2] Hu, Y.L., Qiao, J.L. and Lv, L.X. (2006) Functionalization and Modification of Polyolefins: Science and Technology. Chemical Industry Press, Beijing, 336.

[3] Liang, H.X., Zhang, D.H., Zhang, Y.X., Miao, Z.W. and Yuan, H.L. (2009) Study on Thermo oxidative Aging of Toughening Polyamide and Applied to Automobile, Engineering Plastics Application, 71, 37.

[4] Lian, R.B., Xu, M.Z., Li, Q. and Huang, R. (2008) Study on Properties of Glass Fiber Reinforced Polypropylene Composite, Plastics Science and Technology, 41, 36.

[5] Cui, F.B. and Cao, G.R. (2011) Research on Properties of Glass Fiber Reinforced Polypropylene, Fiber Glass, 9, 1.

[6] Hong, C.K., Kim, M.J., Oh, S.H., Lee, Y.-S. and Nah, C. (2008) Effects of Polypropylene-g-(maleic anhydride/styrene) Compatibilizer on Mechanical and Rheological Properties of Polypropylene/Clay Nanocomposites. Journal of Industrial and Engineering Chemistry, 36, 14. https://doi.org/10.1016/j.jiec.2007.11.001

[7] Ezat, G.S., Kelly, A.L., Mitchell, S.C., Youseffi, M. and Coates, P.D. (2012) Effect of Maleic Anhydride Grafted Polypropylene Compatibilizer on the Morphology and Properties of Polypropylene/Multiwalled Carbon Nanotube Composite. Polymer Composites, 1377, 10.

[8] Lee, J., Kim, J.K. and Son, Y. (2012) Evaluation of Polypropylene Grafted with Maleic Anhydride and Styrene as a Compatibilizer for Polypropylene/Clay Nanocomposites. Polymer Bulletin, 542, 68. https://doi.org/10.1007/s00289-011-0642-5 
[9] Ni, Q.L., Fan, J.Q., Niu, H. and Dong, J.Y. (2011) Enhancement of Graft Yield and Control of Degradation during Polypropylene Maleation in the Presence of Polyfunctional Monomer, Journal of Applied Polymer Science, 2513, 121.

[10] Chen, X.D., Chai, Z.Z., Yang, T., Xiao, J. and Meng, C.M. (2009) Preparation and Application of Odorless and High Grafting Content PP-g-MAH, China Plastics Industry, 63, 37.

[11] Han, W.H., Wu, H.P., Yin, D.S. and Yu, Q. (2009) Thermo Stability of Polypropylene-graft-Maleic Anhydride. China Plastics, 94, 23.

Submit or recommend next manuscript to SCIRP and we will provide best service for you:

Accepting pre-submission inquiries through Email, Facebook, LinkedIn, Twitter, etc. A wide selection of journals (inclusive of 9 subjects, more than 200 journals)

Providing 24-hour high-quality service

User-friendly online submission system

Fair and swift peer-review system

Efficient typesetting and proofreading procedure

Display of the result of downloads and visits, as well as the number of cited articles

Maximum dissemination of your research work

Submit your manuscript at: http://papersubmission.scirp.org/

Or contact msce@scirp.org 